\title{
Transport and Relaxation of Charge in Organic-Inorganic Nanocomposites
}

\author{
I.B. Olenych ${ }^{a, *}$, O.I. Aksimentyeva ${ }^{a}$, B.R. Tsizh ${ }^{b, c}$ And Yu.Yu. Horbenko ${ }^{a}$ \\ ${ }^{a}$ Ivan Franko National University of Lviv, 50, Dragomanov Str., 79005, Lviv, Ukraine \\ ${ }^{b}$ Stepan Gzytsky Lviv National University of Veterinary Medicine and Biotechnologies, \\ 50, Pekarska Str., 79010, Lviv, Ukraine \\ ${ }^{c}$ Kazimierz Wielki University, J.K. Chodkiewicza 30, 85-064 Bydgoszcz, Poland
}

\begin{abstract}
In this work the nanocomposites of poly(3,4-ethylenedioxythiophene) doped with poly(styrenesulfonate) host matrix with porous silicon and $\mathrm{ZnO}$ nanoparticles were manufactured. The charge transport and relaxation processes in organic-inorganic nanocomposites were analyzed based on complex studies of electrical conductivity and depolarization current in the wide temperature range. By means of impedance spectroscopy we have established that increase of content of $\mathrm{ZnO}$ nanoparticles causes the rise in the internal resistance of the hybrid films. Based on the spectra of thermally stimulated depolarization current the localized electron states in the experimental samples are found. The combination of the porous silicon and zinc oxide nanoparticles provides an increase of surface area of the sensors and their high sensitivity to water molecules.
\end{abstract}

DOI: 10.12693/APhysPolA.133.851

PACS/topics: 73.50.-h, 73.61.Ph, 82.35.Np

\section{Introduction}

Hybrid nanocomposites on the base of conjugated polymers reinforced with inorganic nanoparticles of different nature are in the focus of increased attention as composite materials for sensing and optoelectronics applications [1-4]. Typical feature that makes electrically conductive polymers stand out among other high molecular compounds is the conjugated $\pi$-electron bonding system. The conducting polymers reveal electrical conductivity in the doped states though remain insulators when undoped (a neutral state). Due to doping-dedoping processes, the electronic properties of the conjugated polymers can be varied substantially. Protonic acids and electron acceptor substances usually have been used as dopants [5-7]. It has to be noted that conjugated polymers have a capability to be doped not only with donor or acceptor elements, but also with semiconductor nanoparticles.

Increasing the performance of sensory elements based on conjugated polymers, namely, on the poly $(3,4$ ethylenedioxythiophene) abbreviated hereafter as PEDOT is the actual tasks in view of their practical application. One possible solution to the problem is the introduction of semiconductor nanocrystals with high area surface and different types of conductivity into the polymer matrix. In particular, nanocomposites based on the PEDOT and silicon nanoparticles show significant dependence of electrical conductivity on the impurities content as well as on environment factors and radiation $[8,9]$.

*corresponding author; e-mail: iolenych@gmail.com
Silicon nanoclusters can be prepared using a straightforward procedure of electrochemical etching of singlecrystalline silicon, with further formation of a layer of porous silicon (PS) [10, 11]. However, sensitivity and response time of these sensors require some improving.

A special place among the nanosized semiconductors belongs to zinc oxide ( $\mathrm{ZnO}$ ) due to several favorable properties, including good transparency, high electron mobility, wide bandgap, and strong room-temperature luminescence [12-14]. On the other hand, using nanocrystalline $\mathrm{ZnO}$ as gas sensing layer requires high working temperatures $\left(200-300^{\circ} \mathrm{C}\right)$ and special heaters [15]. However, incorporation of $\mathrm{ZnO}$ and silicon nanoclusters give a possibility to use redox properties of conjugated polymers, size effects and large area surface of semiconductor nanoparticles. Thereby it increases the adsorption ability of the sensitive layer and the overall effectiveness of a sensory device.

Present work focuses on the studying the processes of charge transport in nanocomposites based on PEDOT and mixture of PS and $\mathrm{ZnO}$ nanoparticles for sensor electronics. Comprehensive study of the electrical parameters of organic-inorganic nanocomposites showed an improved functionality that extends prospect of practical application of the hybrid materials.

\section{Experiment}

To obtain hybrid films $1.3 \%$ aqueous dispersion of polymeric complex of PEDOT doped with poly(styrenesulfonate) (PSS) was used. A polymeric anion PSS acts simultaneously as an acid dopant and an anionic surfactant which stabilizes dispersion of the polymer $[7,16]$. The other components of hybrid composite were $\mathrm{ZnO}$ and PS nanoparticles. The aqueous polymer 
suspension and $\mathrm{ZnO}$ nanocrystals were purchased from Sigma-Aldrich Co, USA.

The PS nanoparticles were prepared by means of photo-electrochemical etching of single-crystalline silicon $n$-type conductivity $(n-\mathrm{Si})$ with the specific resistance of $4.5 \Omega \mathrm{cm}$. Ethanol solution of hydrofluoric acid (the volume ratio of the components $\mathrm{HF}: \mathrm{C}_{2} \mathrm{H}_{5} \mathrm{OH}=1: 1$ ) was used as an electrolyte. The anodic current density was equal to $30 \mathrm{~mA} / \mathrm{cm}^{2}$ and the etching time was $20 \mathrm{~min}$. Under the technological conditions mentioned above, PS layer with the thickness of about $25 \mu \mathrm{m}$ was formed. After cleaning of samples with distilled water, a resulting porous layer had been taken off from the surface of the plate. It had the shape of a finely-dispersed powder. The silicon particle sizes were ranged from a few tens of $\mathrm{nm}$ to several $\mu \mathrm{m}$.

The resulting powder of $\mathrm{PS}$ was mixed with $\mathrm{ZnO}$ nanoparticles at a volume ratio of $2: 1,1: 1$ and 1:2 to create three series of experimental samples of nanocomposite with different content. The mixtures with a volume of $0.25 \mathrm{ml}$ were added to $0.75 \mathrm{ml}$ PEDOT:PSS suspension and exposed to ultrasonic treatment for $8 \mathrm{~h}$. After this, suspension was deposited to a glass substrate and dried at room temperature for $72 \mathrm{~h}$. Eventually, the monolithic film of PEDOT:PSS-PS-ZnO hybrid composite was obtained. The thickness of the film was about $20 \mu \mathrm{m}$. In order to study electrical properties of the hybrid composites, silver contacts were thermally deposited onto the surfaces of the films. The distance between contacts was $4 \mathrm{~mm}$.

PEDOT:PSS-PS-ZnO hybrid films were characterized by scanning electron microscopy (SEM) REMMA-10202 (Selmi, Ukraine). The electrical properties of the obtained nanocomposites were investigated by means of impedance spectroscopy in the frequency range of $25 \mathrm{~Hz}-$ $1 \mathrm{MHz}$ and thermally stimulated depolarization (TSD) spectroscopy. Impedance spectroscopy of the experimental samples was performed using R, L, C measuring device E7-20 (Kalibr, Belarus) at the room temperature. In the TSD experiment, obtained structures were polarized (polarization voltage was $U=5 \mathrm{~V}$ ) at room temperature and then cooled down to liquid nitrogen temperature in vacuum $\left(10^{-3} \mathrm{~mm} \mathrm{Hg}\right)$. Investigation of the temperature dependence of the depolarization current of preliminarily polarized samples was carried out in the absence of an external electric field at linear heating from 80 to $325 \mathrm{~K}$ at the rate of $0.1 \mathrm{~K} / \mathrm{s}$. The sensory properties of PEDOT:PSS-PS-ZnO films were studied in an airtight chamber, gas medium of which can be changed. The water vapor concentration in air was determined experimentally with a Honeywell HIH-4000-004 humidity sensor.

\section{Results and discussion}

Analysis of the hybrid film's surface was carried out using SEM methods in modes of secondary electrons and energy dispersive X-ray microanalysis (EDXMA). As one can see from Fig. 1, the PEDOT:PSS-PS-ZnO composite formed a monolithic film on the glass substrate.
Study of the film surfaces detects a considerable variation in dispersivity of PS powder in mixture of semiconductor nanoparticles integrated into the polymer films. We found that films with higher content of the PS nanoparticles have a higher surface roughness. Developed surface of the PEDOT:PSS-PS-ZnO films extends the prospect of application of the organic-inorganic composite for gas sensing due to that the increase of the working surface of the touch element enhances its sensitivity.

The chemical composition of hybrid films was determined by the interpretation of energy spectra in X-ray microanalysis mode. Along with the intensive maximum at $1.74 \mathrm{keV}$, which is characteristic for silicon, peaks at 1.05 and $8.65 \mathrm{keV}$ corresponding to zinc atoms were observed. The X-ray microanalysis of the hybrid film structures found the traces of carbon, oxygen and sulfur, which are components of the PEDOT:PSS polymer.

Electrical properties of the PEDOT:PSS-PS-ZnO composite depend on the volume ratio of components. In the case of the random distribution of the disperse phase in the volume of the composite, the charge transfer in the material occurs both through the polymer matrix and through the semiconductor nanoparticles.

Figure 2 presents the Nyquist plots of the PEDOT:PSS-PS-ZnO films in coordinates of the complex plane $Z_{R e}-Z_{I m}$. To interpret the impedance of hybrid films equivalent circuit model was constructed. In this model, the film of organic-inorganic nanocomposites can be regarded as the composition of parallel-connected capacitor with capacitance $C$ and resistor with resistance $R[17,18]$ :

$$
Z(\omega)=\frac{R}{1+\mathrm{j} \omega R C} .
$$

Resistance $R$ is defined both by electrical parameters of the polymer and electrical conductivity of the inorganic nanoparticles. Capacity $C$ is attributed to charge accumulation at interphase boundaries of PEDOT:PSS-PS-ZnO nanocomposite. As it is evident from Fig. 2, the contribution of active resistance of the silicon substrate and the supply contacts is negligibly small (real axis value at $\omega=\infty$ intercept is close to zero). Therefore, we do not consider this resistance in the construction of impedance models for experimental structures. Parameters of the approximation of the impedance spectra of PEDOT:PSS-PS-ZnO films at a volume ratio of $\mathrm{PS}$ and $\mathrm{ZnO}$ components are shown in Table I.

TABLE I

Parameters of the approximation of the impedance spectra of PEDOT:PSS-PS-ZnO hybrid films.

\begin{tabular}{c|c|c}
\hline \hline Volume ratio of & \multicolumn{2}{|c}{ Parameters of approximation } \\
\cline { 2 - 3 } inorganic nanoparticles & resistance $[\mathrm{M} \Omega]$ & capacitance $[\mathrm{pF}]$ \\
\hline PS: $\mathrm{ZnO}=2: 1$ & 20.6 & 4.3 \\
PS: $\mathrm{ZnO}=1: 1$ & 43.8 & 3.9 \\
PS: $\mathrm{ZnO}=1: 2$ & 69.6 & 5.5
\end{tabular}



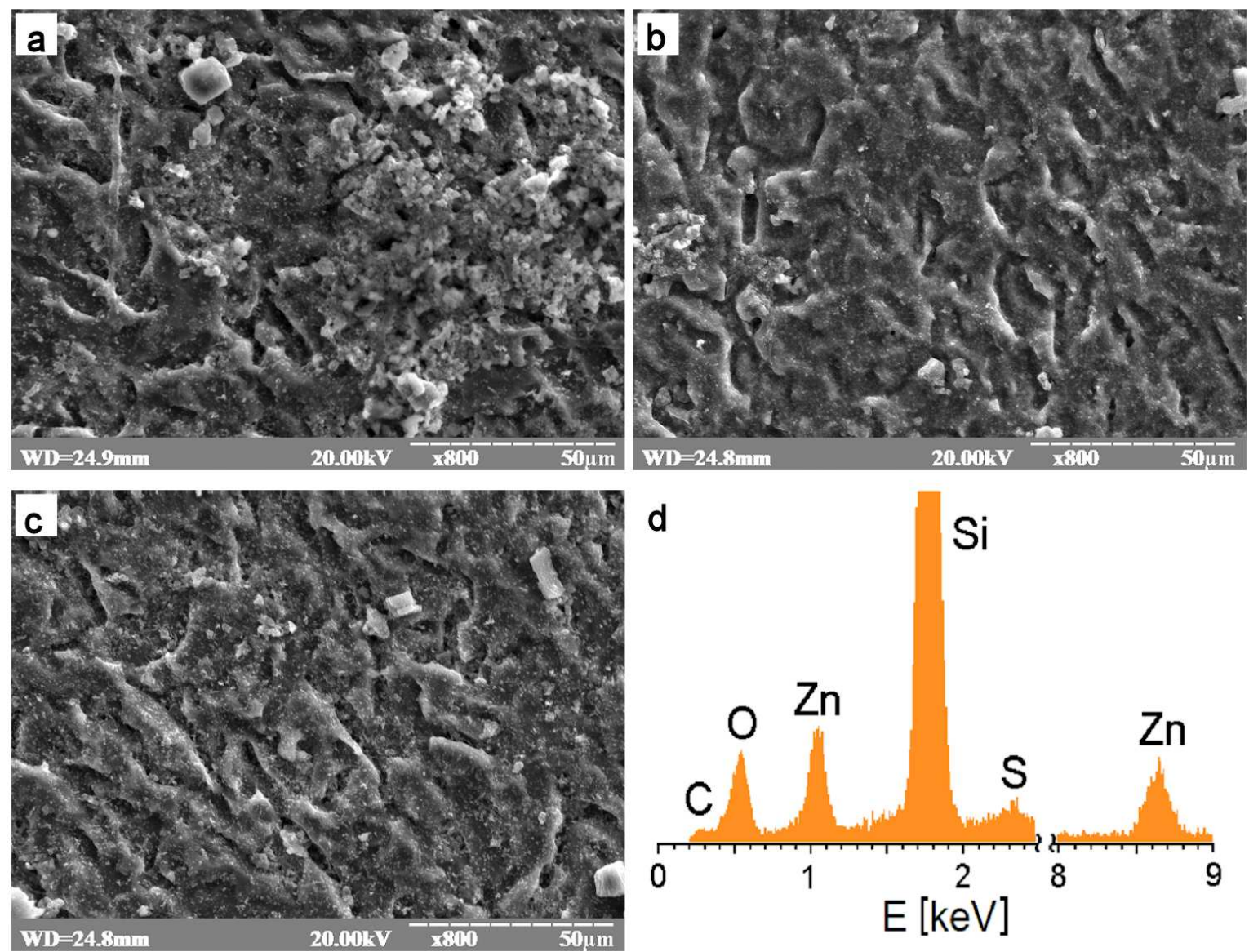

Fig. 1. SEM images of the surfaces of PEDOT:PSS-PS-ZnO films at a volume ratio of components PS: ZnO = 2:1 (a), PS: $\mathrm{ZnO}=1: 1$ (b), and PS: $\mathrm{ZnO}=1: 2$ (c). X-ray microanalysis of PEDOT:PSS-PS-ZnO composite (d).

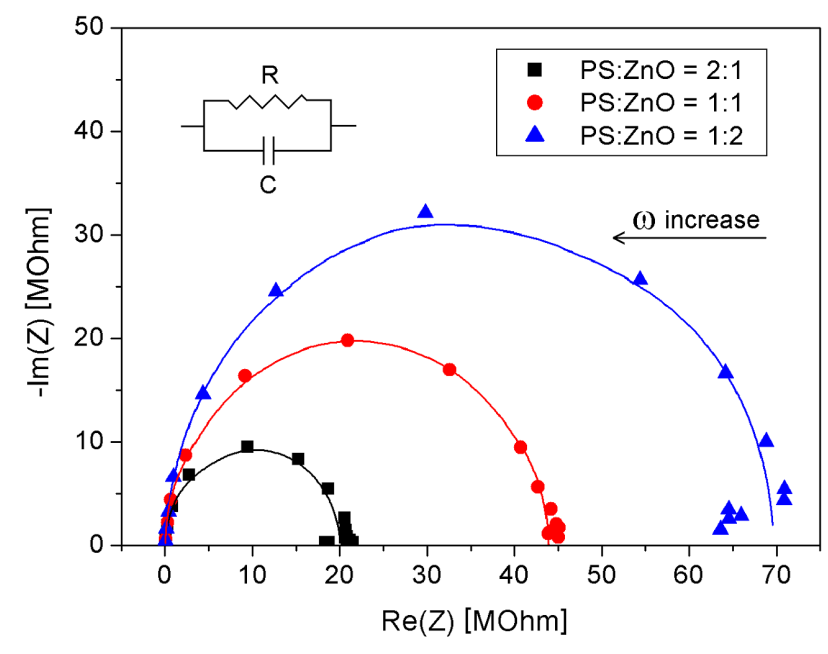

Fig. 2. Nyquist plots of the PEDOT:PSS-PS-ZnO films. Inset: equivalent circuit diagram of the experimental samples.

In accordance with $R-C$ model of experimental samples increase of content of $\mathrm{ZnO}$ nanoparticles caused the rise in the internal resistance of the PEDOT:PSS-PS$\mathrm{ZnO}$ films from 20 to $70 \mathrm{M} \Omega$. This may be caused by a higher resistance of wide band $\mathrm{ZnO}$ nanoparticles com- pared with resistance of PS. In addition, we observed a significant reduction in the hybrid films resistance in the $0.1-1 \mathrm{MHz}$ frequency range. Electric capacitance of hybrid composite is less dependent on its composition than resistance. Observed impedance spectra can be governed by charge relaxation at phase boundaries which is characteristic for multicomponent media [19]. Relaxation time for such processes depends on electrical parameters of the composite components, therefore the change of the volume ratio of inorganic nanoparticles causes the variation of time constants for parallel $R-C$ circuits that are used to interpret PEDOT:PSS-PS-ZnO films impedance spectra.

Studies of the relaxation processes in the hybrid nanocomposites were performed using TSD spectroscopy in 80-325 $\mathrm{K}$ temperature range. In the case of disordered systems the trap levels are distributed quasi-continuously on the activation energy $[20,21]$. To determine the activation energy of electrically active defects in experimental films, the temperature dependences of the depolarization current have been measured. The TSD spectra of the PEDOT:PSS-PS-ZnO films at different volume ratio of nanocomponents were very similar (Fig. 3). This indicates the similar activation energy distribution of electrically active defects in experimental samples. 

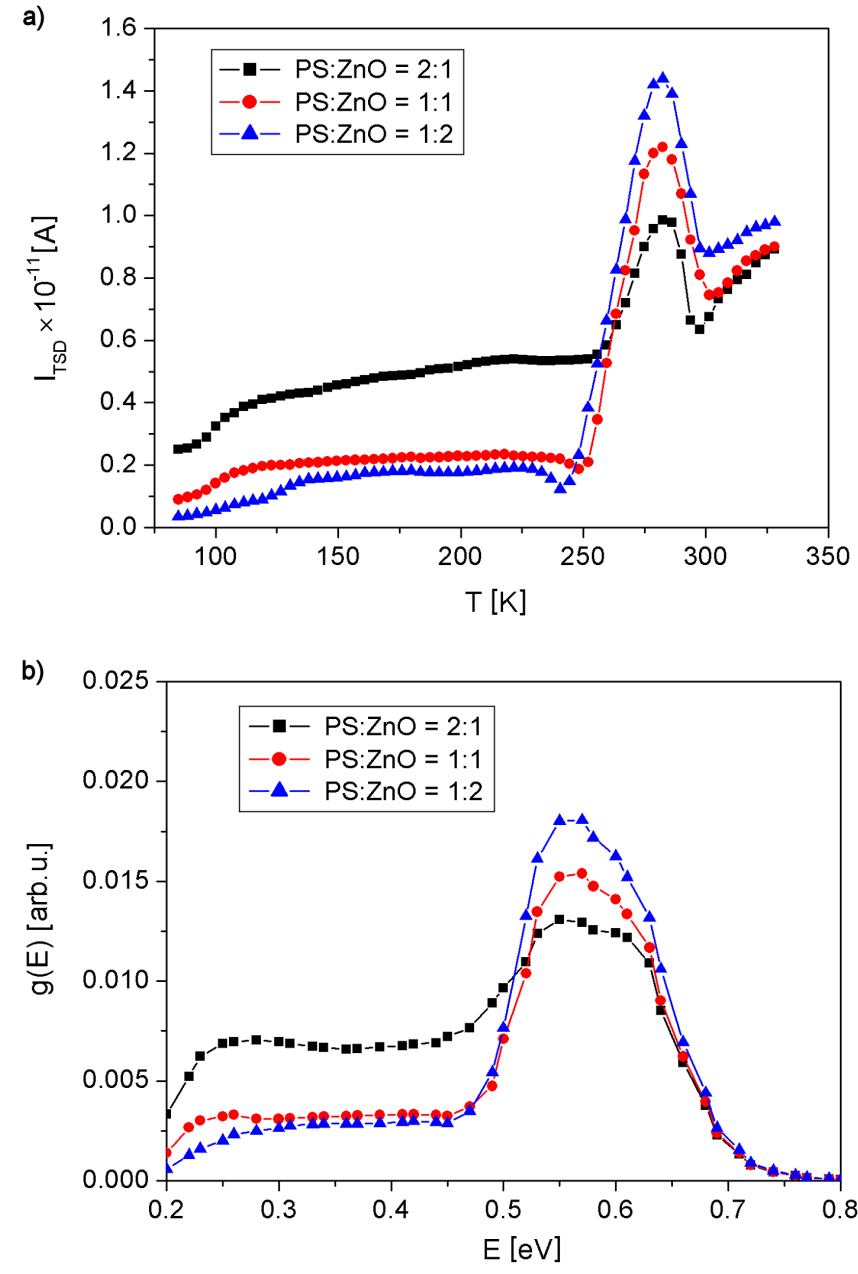

Fig. 3. Temperature dependences of depolarization current (a) and energy distribution of the density of states (b) in the PEDOT:PSS-PS-ZnO films.

The analysis of the TSD spectra and the calculation of the density of states energy distribution suggests that the groups of trap levels, with differences in nature and activation energies in the ranges of $0.2-0.3,0.5-0.55$, and $0.6-0.65 \mathrm{eV}$ are present. It is likely that the bands at 0.2 0.3 and $0.6-0.65 \mathrm{eV}$ are associated with the electrically active defects in the PS nanoparticles [21]. The band at $0.5-0.55 \mathrm{eV}$ is probably related to $\mathrm{ZnO}$ nanocrystals, because band intensity increases for samples with more content of $\mathrm{ZnO}$.

We have found experimentally that the electrical characteristics of composite films are strongly dependent on the surrounding atmosphere. In particular, increasing relative humidity results in significant decrease of the electrical resistance of the hybrid PEDOT:PSS-PS-ZnO films (see Fig. 4).

The obtained dependences were caused by an interaction of $\mathrm{H}_{2} \mathrm{O}$ polar molecules with the surface of PEDOT:PSS-PS-ZnO composite. It changes the polymer conductivity and electronic parameters of semicon-

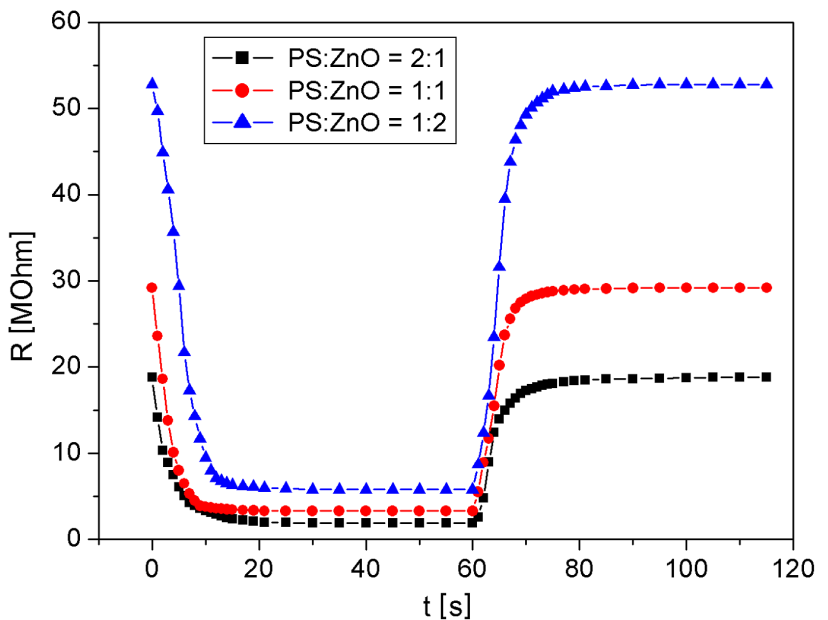

Fig. 4. Responses of resistance of the sensors based on PEDOT:PSS-PS-ZnO films to the pulse of relative air humidity.

ductor nanocrystals by adsorption-electric effects [22, 23]. The interaction with the water vapor has a character of physical adsorption, because the initial conductivity of the hybrid films is restored after scavenging and pumping off the water vapor from the experimental chamber. The time of response of the sensory elements to changing gas concentration is about $20 \mathrm{~s}$. When compared with the moisture-sensitive structures PS-silicon substrate [24] and thin-film humidity sensor based on PEDOT:PSS doped with PS powder and carbon nanotubes [8], the response of our hybrid film sensors is faster.

\section{Conclusions}

We have prepared PEDOT:PSS-PS-ZnO composite films for gas sensing. Forming of monolithic hybrid film has been confirmed by scanning electron microscopy. Electrical parameters of the obtained composites depend on the volume ratio of inorganic components. The increase of content of $\mathrm{ZnO}$ nanoparticles causes the rise in the internal resistance of the hybrid films from 20 to $70 \mathrm{M} \Omega$. As follows from the analysis of TSD spectra of hybrid films, the energy distribution of the density of states for nonequilibrium carriers shows peaks in the ranges of $0.2-0.3,0.5-0.55$, and $0.6-0.65 \mathrm{eV}$. Such localized electron states influence the charge transport in the PEDOT:PSS-PS-ZnO composites.

It has been revealed experimentally that the adsorption of water molecules changes electrical properties of obtained composites under test. The combination of the porous silicon and zinc oxide nanoparticles provides an increase of surface area of the sensors and their high sensitivity to water molecules. The kinetics of the resistance response of the hybrid composites PEDOT:PSS-PS-ZnO to the change of water vapor concentration is fast enough to be employed in microelectronic humidity sensors. 


\section{References}

[1] R. Francis, N. Joy, P. Aparna, R. Vijayan, Polym. Rev. 54, 268 (2014).

[2] B.R. Tsizh, O.I. Aksimentyeva, M.I. Chokhan, Yu.R. Portak, Mol. Cryst. Liq. Cryst. 535, 220 (2011).

[3] E. Holder, N. Tessler, A.L. Rogach, J. Mater. Chem. 18, 1064 (2008).

[4] I. Karbovnyk, I. Olenych, O. Aksimentyeva, H. Klym, O. Dzendzelyuk, Yu. Olenych, O. Hrushetska, Nanoscale Res. Lett. 11, 84 (2016).

[5] A.J. Heeger, Synthet. Met. 125, 23 (2001).

[6] Ph. Leclere, M. Surin, P. Brocorens, M. Cavallini, F. Biscarini, R. Lazzaroni, Mater. Sci. Eng. R Rep. 55, 1 (2006).

[7] A.V. Kubarkov, O.A. Pyshkina, V.G. Sergeyev, Polym. Sci. Ser. B 56, 360 (2014).

[8] I.B. Olenych, O.I. Aksimentyeva, L.S. Monastyrskii, Yu.Yu. Horbenko, L.I. Yarytska, Nanoscale Res. Lett. 10, 187 (2015)

[9] I.B. Olenych, O.I. Aksimentyeva, L.S. Monastyrskii, O.S. Dzendzeliuk, Mol. Cryst. Liq. Cryst. 640, 165 (2016).

[10] H. Föll, M. Christophersen, J. Carstensen, G. Hasse, Mater. Sci. Eng. R Rep. 39, 93 (2002).

[11] O. Bisi, S. Ossicini, L. Pavesi, Surf. Sci. Rep. 38, 1 (2000).

[12] A. Moezzi, A.M. McDonagh, M.B. Cortie, Chem. Eng. J. 185-186, 1 (2012).
[13] Z.L. Wang, J. Phys. Condens. Matter 16, R829 (2004).

[14] Y.W. Heo, D.P. Norton, L.C. Tien, Y. Kwon, B.S. Kang, F. Ren, S.J. Pearton, J.R. LaRoche, Mater. Sci. Eng. R Rep. 47, 1 (2004).

[15] B.R. Tsizh, O.I. Aksimentyeva, V.Y. Lazorenko, M.I. Chokhan, Solid State Phenom. 200, 305 (2013).

[16] J. Ouyang, Q. Xu, C.-W. Chu, Y. Yang, G. Li, J. Shinar, Polymer 45, 8443 (2004).

[17] L.K. Pan, H.T. Huang, C.Q. Sun, J. Appl. Phys. 94 2695 (2003)

[18] S. Bellucci, I. Bolesta, I. Karbovnyk, R. Hrytskiv, G. Fafilek, A.I. Popov, J. Phys. Condens. Matter 20, 474211 (2008).

[19] Y.P. Emets, Techn. Phys. 48, 317 (2003).

[20] Y. Gorohovatsky, H. Bordovsky, Thermally Activational Current Spectroscopy of Highresistance Semiconductors and Dielectrics, Nauka, Moscow 1991.

[21] I. Olenych, B. Tsizh, L. Monastyrskii, O. Aksimentyeva, B. Sokolovskii, Solid State Phenom. 230, 127 (2015)

[22] M. Kuş, S. Okur, Sens. Actuat. B 143, 177 (2009).

[23] N. Rastgar, D.J. Rowe, R.J. Anthony, B.A. Merritt, U.R. Kortshagen, E.S. Aydil, J. Phys. Chem. C 117, 4211 (2013)

[24] I.B. Olenych, L.S. Monastyrskii, O.I. Aksimentyeva, B.S. Sokolovskii, Ukr. J. Phys. 56, 1198 (2011). 